\title{
Invited Response on: Perceptions of Silicone Structure and Function
}

\author{
Richard Zinn $^{1} \cdot$ Sibon K. Fuzzard ${ }^{1} \cdot$ Rodrigo Teixeira ${ }^{1,2}$
}

Received: 7 April 2020/Accepted: 8 April 2020/Published online: 23 April 2020

(C) Springer Science+Business Media, LLC, part of Springer Nature and International Society of Aesthetic Plastic Surgery 2020

Level of Evidence $V$ This journal requires that authors assign a level of evidence to each article. For a full description of these Evidence-Based Medicine ratings, please refer to the Table of Contents or the online Instructions to Authors www.springer.com/00266.

\section{Dear Colleague,}

The author of this article is applauded in identifying the fact that 'silicone' implants are not all chemically the same and therefore cannot be compared. Certainly, this factor should be looked at more closely in future literature. Some polymers may cause toxic and allergic reactions while others may be inert as we have always believed. Further chemicals added to the production phase may also be a cause of the symptoms we attribute to Silicone Implant Illness (SII).

There is certainly scope for a study looking at categorizing the exact chemical contents of all breast implants on the market. Furthermore, there is scope for a study to look at brands of silicone implants implicated in SII to determine if specific implant brands cause this problem more than others. A new area of research awaits.

We agree with the author that breast implant companies may be less than eager to contribute such data willingly to be studied. Being implicated in another implant-related disease such as BIA-ALCL would spell ruin for a breast mplant company. We have seen this scenario play out recently for BIA-ALCL. This also could have implications as the author suggests for liability.

Another way SII and the associated risk factors will be able to be tracked is through breast registries. As the data from these breast registries mature, trends will become visible. The range of complications of breast surgery with implants and the risk factors for these complications will be easily elucidated. Being collected prospectively, these data may shed more light on whether some brands are more likely to cause breast implant illness than others. In the meantime, testing the chemical contents of all silicone implants on the market is a logical next step.

\section{Compliance with Ethical Standards}

Conflict of interest The authors disclosure their prior publication on the topic, and declare they have no conflict of interest.

Human and Animal Rights This article does not contain any studies with human participants or animals performed by any of the authors.

Informed Consent For this type of study informed consent is not required.

Publisher's Note Springer Nature remains neutral with regard to jurisdictional claims in published maps and institutional affiliations.
Sibon K. Fuzzard

sibon_fuzzard@hotmail.com

1 Department of Plastic and Reconstructive Surgery, Northern Health Melbourne, 185 Cooper Street, Epping, VIC 3076, Australia

2 The University of Melbourne Medical School, Grattan Street, Parkville, VIC 3010, Australia 